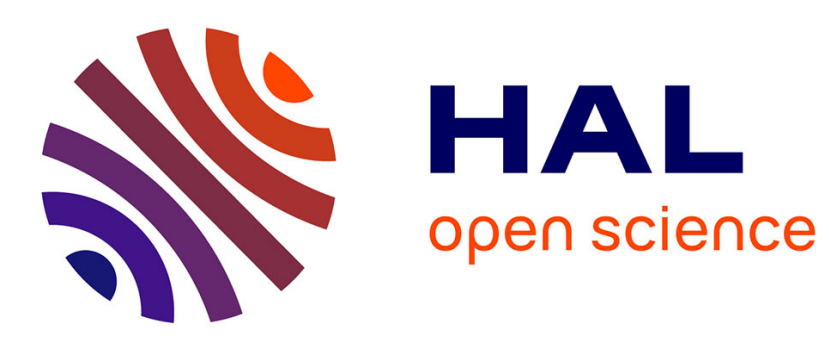

\title{
Staging for locally advanced pancreatic cancer
}

M.J.M. Morak, J.J. Hermans, H.G. Smeenk, W.M. Renders, J.J.M.E.

Nuyttens, G. Kazemier, C.H.J. van Eijck

\section{To cite this version:}

M.J.M. Morak, J.J. Hermans, H.G. Smeenk, W.M. Renders, J.J.M.E. Nuyttens, et al.. Staging for locally advanced pancreatic cancer. EJSO - European Journal of Surgical Oncology, 2009, 35 (9), pp.963. 10.1016/j.ejso.2009.01.013 . hal-00556275

\section{HAL Id: hal-00556275 \\ https://hal.science/hal-00556275}

Submitted on 16 Jan 2011

HAL is a multi-disciplinary open access archive for the deposit and dissemination of scientific research documents, whether they are published or not. The documents may come from teaching and research institutions in France or abroad, or from public or private research centers.
L'archive ouverte pluridisciplinaire HAL, est destinée au dépôt et à la diffusion de documents scientifiques de niveau recherche, publiés ou non, émanant des établissements d'enseignement et de recherche français ou étrangers, des laboratoires publics ou privés. 


\section{Accepted Manuscript}

Title: Staging for locally advanced pancreatic cancer

Authors: M.J.M. Morak, J.J. Hermans, H.G. Smeenk, W.M. Renders, J.J.M.E. Nuyttens, G. Kazemier, C.H.J. van Eijck

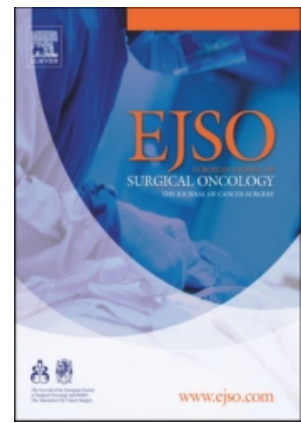

PII: $\quad$ S0748-7983(09)00031-6

DOI: $\quad$ 10.1016/j.ejso.2009.01.013

Reference: $\quad$ YEJSO 2786

To appear in: European Journal of Surgical Oncology

Received Date: 23 September 2008

Revised Date: 27 January 2009

Accepted Date: 28 January 2009

Please cite this article as: Morak MJM, Hermans JJ, Smeenk HG, Renders WM, Nuyttens JJME, Kazemier G, van Eijck CHJ. Staging for locally advanced pancreatic cancer, European Journal of Surgical Oncology (2009), doi: 10.1016/j.ejso.2009.01.013

This is a PDF file of an unedited manuscript that has been accepted for publication. As a service to our customers we are providing this early version of the manuscript. The manuscript will undergo copyediting, typesetting, and review of the resulting proof before it is published in its final form. Please note that during the production process errors may be discovered which could affect the content, and all legal disclaimers that apply to the journal pertain. 


\section{STAGING FOR LOCALLY ADVANCED PANCREATIC CANCER}

M.J.M. Morak ${ }^{1}$, J.J. Hermans ${ }^{2}$, H.G. Smeenk ${ }^{1}$, W.M. Renders ${ }^{1}$, J.J.M.E. Nuyttens ${ }^{3}$, G. Kazemier $^{1}$ and C.H.J. van Eijck ${ }^{1}$

${ }^{1}$ Department of Surgery

Erasmus Medical Centre, Rotterdam, The Netherlands

${ }^{2}$ Department of Radiology

Erasmus Medical Centre, Rotterdam, The Netherlands

${ }^{3}$ Department of Radiotherapy

Erasmus Medical Centre, Rotterdam, The Netherlands

Correspondence to:

C.H.J. van Eijck, MD, PhD

Department of Surgery

Erasmus Medical Centre Rotterdam

's Gravendijkwal 230

3015 CE Rotterdam,

The Netherlands

Tel: +31-107033854

Mail: c.vaneijck@erasmusmc.nl

Original article 


\section{Abstract}

Aim: To address the role of a dedicated radiologist and high quality CT scanning in staging of patients referred with suspected locally advanced pancreatic cancer. Furthermore, the value of laparoscopy in detecting CT occult metastases in these patients was assessed.

Methods: In a prospective cohort study, 116 patients with suspected unresectable pancreatic cancer referred from peripheral hospitals (107) or our own gastroenterology department (9) were analysed. CT scans from referral centres were reviewed and in case of locally advanced disease or uncertain metastatic disease, patients underwent a laparoscopy to detect CT occult metastases. Patients without metastases were offered 5-FU based chemoradiotherapy.

Results: After reviewing 107 abdominal CT scans from referral centres, 73 (68\%) scans had to be repeated due to unacceptable quality. Locally advanced disease was confirmed in 59 (55\%) patients and metastatic disease was found in 24 patients (22\%). During laparoscopy, metastases were found in 24/ 68 (35\%) patients with locally advanced disease on CT scan and metastases were confirmed in 3/5 (60\%) with suspected metastases.

Overall, only 46/ 116 (40\%) patients with suspected unresectable disease appeared to have locally advanced pancreatic cancer after adequate staging including laparoscopy in our centre. Conclusion: Correct staging is difficult in patients with suspected locally advanced pancreatic cancer and should preferably be performed in centres with technically advanced equipment and experienced radiologists. Laparoscopy should be offered to patients before locoregional therapy.

Key Words: locally advanced pancreatic cancer $\downarrow$ accurate staging $\downarrow$ diagnostic laparoscopy chemoradiotherapy 


\section{Introduction}

Pancreatic carcinoma is the 4th most common cause of cancer related death worldwide.[1] Despite improved quality of surgery, the prognosis of patients with pancreatic cancer is still dismal. Additional treatment seems essential to improve outcome in these patients. Several studies have shown benefit for neoadjuvant or adjuvant treatment in patients with resectable pancreatic cancer.[2-4] In contrast, 80-90\% of patients suffer from either unresectable or metastatic disease at the time of diagnosis. In these patients, no curation can be established and survival relies on the effectiveness of palliative chemotherapy and/ or radiation.

The introduction of spiral or helical computed tomography (CT) scanning has greatly affected the selection of patients eligible for surgical exploration, thus increasing overall resectability rates in these patients. Vascular tumour ingrowth for example, can be visualised with preoperative helical scanning with high accuracy.[5] However, small metastatic lesions can be missed.[6-8]

Laparoscopy has proven to be able to detect these metastases that mainly consist of peritoneal and superficial liver lesions. As a result several groups recommend diagnostic laparoscopy in combination with or without laparoscopic ultrasonography as a standard procedure in the staging of all patients with resectable as well as unresectable pancreatic cancer.[9, 10] However recent studies using high quality CT-scanning in resectable pancreatic cancer patients revealed that only a small percentage of these patients would benefit from a routine diagnostic laparoscopy.[11, 12] Nieveen et al. found a 13\% detection rate of metastatic disease during laparoscopy in a group of 297 consecutive patients with resectable disease. The low detection rate and subsequent need for, and excellent long term results of, bypass surgery validate the conclusion that general use of diagnostic laparoscopy in all pancreatic cancer patients is not cost-effective and not justified.[12] Tilleman et al. concluded that there is no 
indication to perform a routine laparoscopy in patients with resectable pancreatic cancer, but that there might be an indication in a, non-specified, well-defined high-risk subgroup.[11]

Patients with locally advanced disease in the work-up, are known to have this higher incidence of CT-occult liver and peritoneal metastases[13] and laparoscopy has been reported to be of additional value to improve assessment of tumour stage in these patients with metastatic detection rates varying between 34-37\%.[6, 14, 15]

Because a laparoscopy appears most valuable in patients with locally unresectable but not in resectable disease, cautious staging based on CT scanning is of utmost importance. However, patients with pancreatic cancer often present in non-specialised centres without high quality CT scans or specific pancreatic imaging protocols. After referral to specialised centres, the quality of the CT scans has to be assessed and designated radiologists review appropriate scans. New scans are made in case of poor quality.

Several studies imply that chemotherapy should be offered to patients with locally advanced pancreatic cancer as in metastatic disease because of disappointing results of chemoradiotherapy. However, laparoscopy was no part of the staging regime in these studies resulting in the inclusion of metastasised disease and thus, considering the fact that chemoradiotherapy only has an effect on localised disease, an underestimation of the effect of chemoradiotherapy.[16-18] Based on previous results in our centre, chemoradiotherapy is a promising option in patients with unresectable, non-metastasised pancreatic cancer based on laparoscopy.[19]

The aim of this study was to address the role of a dedicated radiologist and high quality CT scanning in staging of patients referred with suspected locally advanced pancreatic cancer. Furthermore, the value of laparoscopy in detecting CT occult metastases in these patients was assessed. 


\section{Methods}

In this study we evaluated all patients referred to the surgical department of the Erasmus Medical Centre (Erasmus MC) between January 1995 and March 2007 with the diagnosis of locally advanced pancreatic cancer based on abdominal CT scan. In most patients with obstructive jaundice, preoperative biliary drainage was performed by endoscopic retrograde cholangiopancreatography (ERCP) and placement of an endoprothesis; others received either a papillotomy or percutaneous transhepatic drainage.

\section{Abdominal CT Scan}

From 1995- 1999 CT scanning in the Erasmus MC was performed using a Siemens Somatom

Plus single slice CT (Siemens Medical Solutions; Erlangen, Germany). After 1999 a Siemens multislice spiral CT scanner was used (Siemens Medical Solutions; Erlangen, Germany). Our standard triphasic pancreatic protocol consists of a non contrast enhanced scan with a slice thickness of $5.0 \mathrm{~mm}$, an arterial/ pancreatic phase at 40 seconds after the initiation of contrast injection (Visipaque $320 \mathrm{mg} / \mathrm{ml}$ ) with a slice thickness of $3.0 \mathrm{~mm}$, followed by 5.0mm-thick slices at 80 seconds (portal venous phase). One hundred fifty milliliters of contrast material is injected intravenously at a rate of $3.5 \mathrm{ml} / \mathrm{sec}$.

One of our designated radiologists (Hermans) reviewed all abdominal CT scans. CT scans from peripheral hospitals were judged as of sufficient quality in case of triphasic scanning, a comparable scanning protocol, a maximum slice thickness of $3.0 \mathrm{~mm}$ in arterial/ pancreatic phase and $5.0 \mathrm{~mm}$ in portal venous phase and no movement artefacts.

A pancreatic tumour was classified as resectable, uncertain resectable, unresectable, suspected metastatic or metastatic, based on CT scan. In case of metastatic disease, the resectability of the primary tumour was not addressed and could be either resectable or unresectable. All metastatic lesions were histopathologically proven. 
In case of (uncertain) resectable disease, a laparotomy was performed outside the perspectives of this study. All patients with unresectable disease and uncertain metastatic disease underwent a laparoscopy.

Unresectable disease was described by Loyer and Phoa defined as: i) infiltration of peripancreatic fatplanes, the hepatoduodenal ligament and the mesentery (Figure I), ii) $>180^{\circ}$ encasement of the portal or superior mesenteric vein or of the hepatic or superior mesenteric artery (Figure I), iii) infiltration of the vessel, i.e. ingrowth or thrombosis (Figure II).[20, 21]

\section{Diagnostic Laparoscopy}

Patients undergo examination under general anaesthesia as outpatients or with a 24-hour observation stay. Following establishment of pneumoperitoneum, a $10 \mathrm{~mm}$ trocar is inserted through an infraumbilical incision. The laparoscope is introduced through this trocar, and examination begins by inspection of the lower abdomen and pelvis. The laparoscope is then rotated for examination of the upper quadrants. Inspection begins by assessment of the omentum and subdiaphragmatic spaces. Meticulous examination of the liver surface is essential. Insertion of a second and third 5-mm trocar in the right upper quadrant is necessary for adequate evaluation of the posterior aspect of the liver and biopsies of suspected lesions. Elevation of the transverse colon and inspection of the mesentery and Treitz' ligament is performed with the patient placed in anti- Trendelenburg position. Finally biopsies of suspected lesions are taken with biopsy forceps (superficial lesions) or with Rotex or Tru-cut needles (deeper lesions) under guidance of the laparoscope. We do not perform extensive dissection of the retrogastric space and lesser sac routinely, nor peritoneal lavage.

Laparoscopic ultrasound was not used routinely.

\section{Treatment}

If no metastases were found during laparoscopy, a percutaneous or endoscopic ultrasound fine needle aspiration of the primary tumour was performed to confirm malignancy. 
All patients with true (laparoscopy based, histopathologically proven) locally advanced, i.e. non metastasised, pancreatic cancer were offered chemoradiotherapy consisting of 5-FU in combination with radiotherapy.

Patients with metastatic disease based on laparoscopy were offered chemotherapy.

\section{Statistics}

Analysis was done with the Statistical Package for the Social Sciences version 11.5 (SPSS). Survival curves were computed according to the Kaplan Meier method and comparison of survival curves was done with the log-rank test. 


\section{Results}

From January 1995 until March 2007116 consecutive patients referred with the presumed diagnosis of locally advanced pancreatic tumours according to CT scan were incorporated in this prospective cohort study. The median age in these patients was 63 years (range 43-92 years), and 68 of the patients were men. Presenting symptoms were weight loss ( $n=94)$, pain ( $n=87)$, obstructive jaundice $(n=68)$ and diabetes mellitus de novo $(n=13)$. Ca 19.9 was elevated (>34kU/l) in 102 patients, whereas CEA was elevated $(>4.99 \mu \mathrm{g} / \mathrm{l})$ in 56 patients. Tumour size was larger than $3 \mathrm{~cm}$ radiologically in 95 patients.

\section{CT Scan}

Of the 116 patients, 107 patients were referred from peripheral hospitals, while 9 patients were referred from our own gastroenterology department. CT scans of seventy-three (68\%) of the 107 referred patients had to be repeated while the other scans were of predefined quality. Unresectable disease was found in 59 patients and uncertain metastatic disease in 5 patients. Furthermore, resectable disease was found in 6 patients, uncertain unresectable disease in 13 patients and metastatic disease in 24 patients.

The cause of unresectability in all 68 (59 plus the nine patients from our own gastroenterology department) patients is shown in Table I.

\section{Diagnostic Laparoscopy}

The mean amount of days between CT scan and laparoscopy was 13 (range 0- 49 days, SD \pm 10 ). The median operative time, including anaesthesia, was 79 minutes (range 41- $130 \mathrm{~min}$ ). In two patients there was an indication for a consecutive laparoscopic gastroenterostomy. Twenty-four (35\%) of the 68 patients with unresectable disease appeared to have metastatic disease at laparoscopy. Metastases were mainly located on the liver and peritoneum (Table II). No correlation could be found between the reason for unresectability (i.e. ingrowth into peripancreatic fatplane, into adjacent vessels or encasement of these vessels) on CT scan and 
the presence of metastases at laparoscopy. There was no difference in detection rate between CT scans performed before 1999 (6/16=27\%) and CT scans performed in/or after 1999 (18/28=39\%). All metastases were proven histopathologically.

There were 2 major complications in the entire group: In one patient the laparoscopic gastroenterostomy was insufficient for which a relaparotomy was necessary and in another patient a prolonged paralytic ileus was seen after laparoscopy. The mean overall hospital stay was 5 days (range 1- 50 days).

Of the 5 patients with suspected metastatic lesions based on CT scan, all lesions were located in the liver. Laparoscopy confirmed these metastases in only three of the patients. After laparoscopy 44 (65\%) of the 68 patients with locally advanced pancreatic cancer on abdominal CT scan were diagnosed with true, non-metastatic, locally advanced disease and two (40\%) out of five patients with uncertain metastatic disease. A percutaneous biopsy or fine needle aspiration during subsequent endoscopic ultrasound confirmed malignancy in all these patients.

In conclusion, after reviewing abdominal CT scan and laparoscopy in all 116 patients with suspected unresectable disease at referral, 46/ 116 patients (40\%) appeared to have nonmetastatic unresectable pancreatic cancer and chemoradiotherapy was offered to all these patients. 


\section{Discussion}

\section{Summary}

Because the complexity of pancreatic cancer, the different stages it presents in, the need for exact imaging, the difficulty of surgery and the ever changing non-surgical or adjuvant treatments, it remains a disease best treated in specialised centres. Although for diagnosing pancreatic cancer abdominal CT scan remains an important imaging module, the quality of scans performed in non-specialised centres remains poor and a high quality pancreatic cancer specified protocol CT scan needs to be performed to be able to draw conclusions concerning resectability and the presence of metastases. On the other hand radiologists need to be trained to be able to address pancreatic cancer specific issues, as there are vascular invasion and or encasement. This is based on our results where CT scans had to be repeated in our institution in $68 \%$ of the patients and only $55 \%$ of the patients referred for unresectable disease based on CT scan, did have an unresectable pancreatic tumour after reviewing. And even our staging methods and radiologist’s experience are not flawless. Although we diagnosed locally advanced disease on abdominal CT scan and laparoscopy in 46 patients (i.e. only $40 \%$ of the originally referred patients), a laparotomy and thoracic CT scan revealed metastases in six more patients. Because a thoracic CT scan is less invasive than a laparoscopy, a thoracic CT scan before laparoscopy should be considered in future studies on unresectable pancreatic cancer.

\section{Literature}

In this second largest group of patients reported in literature with locally advanced pancreatic cancer that underwent a laparoscopy we can confirm the 37\% detection rate of CT occult metastases found by Shoup et al.[14] However, where they used a single site for the laparoscope and only inserted a 5-mm trocar in case of suspected metastatic disease, we routinely inserted a second and third 5-mm trocar in the right upper quadrant for adequate 
evaluation of the posterior aspect of the liver. Consequently, the 37\% detection rate after laparoscopy mentioned by Shoup et al. might even be an underestimation. One smaller study performed by Liu found similar results (34\% detection rate), but metastases were mainly found through peritoneal lavage cytology during laparoscopy.[6] We did not perform peritoneal lavage cytology. A combination of standard laparoscopy and peritoneal lavage might have led to an even higher detection rate.

\section{Locally advanced vs. metastatic disease}

In our opinion it is important to discriminate between locally advanced and metastatic disease because of the implications for further treatment. In the past, locally advanced and metastatic pancreatic cancer was described as one disease entity, i.e. advanced pancreatic cancer, and was consequently treated identical. In this combined group, chemotherapy containing gemcitabine is the better treatment option although, as far as we know, randomised controlled trials containing radiotherapy have not been performed.[22-24]

Locally advanced and metastatic disease should be considered as two different entities. Where the optimal treatment, i.e. chemotherapy or chemoradiotherapy, is still disputable in locally advanced cancer, chemotherapy is the only option for patients with metastasised disease. Overall survival figures in locally advanced disease vary between 11- 14 months after chemotherapy[25, 26] and between 7- 15 months after chemoradiotherapy depending on the used chemotherapeutics.[16, 17, 19, 26] In metastatic disease, median survival figures vary between 8- 11 months.

The staging however, remains inadequate because these studies are based on CT imaging only, consequently incorporating metastatic disease in over a third of the included patients. This resulted in underestimating the effect of locoregional therapy, i.e. chemoradiotherapy, in favour of systemic therapy, which proved to be effective in metastatic disease. 
In two studies, patients with locally advanced pancreatic cancer were adequately staged.[27, 28] Both studies addressed the effect of chemoradiation either in combination with 5-FU and mitomycin-C or with gemcitabine and irinotecan. Cohen et al. reported a median survival of $8.4 v s .7 .1$ months after radiotherapy with 5-FU and mitomycin-C compared to radiotherapy alone. Mishra et al. preliminary stopped their study after accruing 20 patients because of disappointing results (median survival of 8.8 months). However both studies used a laparotomy as staging module: Cohen staged all patients with laparotomy and Mishra staged at least part of his patients with laparotomy. Their poor results might be explained by the fact that major operative trauma may favour the development of tumour recurrence. An invasiveness dependent association between surgical trauma and tumour recurrence has been supported by previous in vivo and in vitro studies, showing less metastases after laparoscopy compared to laparotomy.[29, 30] In our study all patients were staged by laparoscopy, resulting in less surgical stress and indeed a better median overall survival (11.7 months).

\section{Conclusion}

In conclusion we think that adequate staging should be performed in a specialised centre because of the considerable percentage of incorrectly staged patients referred from peripheral centres. Our study shows that after reviewing CT scans and laparoscopy in patients with suspected locally advanced pancreatic cancer, only $40 \%$ of these patients appeared to have true unresectable, non-metastasised pancreatic cancer. Only after careful staging including laparoscopy, results of future studies on either locoregional therapy or systemic therapy for locally advanced pancreatic cancer can be interpreted correctly.

\section{Conflict of interest}

The authors state that they have no conflict of interest. 


\begin{tabular}{|l|c|}
\hline Table I: CT criteria for unresectability (n=68) & \\
\hline Infiltration of peripancreatic fatplanes, HDL +/- mesentery & $\mathrm{N}=1$ \\
\hline$>180^{\circ}$ encasement of the PV, SMV, HA or SMA & $\mathrm{N}=38$ \\
\hline Infiltration of the vessel (i.e. ingrowth or thrombosis) & $\mathrm{N}=16$ \\
\hline Combination of encasement and infiltration of the vessel & $\mathrm{N}=13$ \\
\hline
\end{tabular}

$\mathrm{HDL}=$ hepatoduodenal ligament, $\mathrm{PV}=$ portal vein, $\mathrm{SMV}=$ superior mesenteric vein, $\mathrm{HA}=$ hepatic artery, SMA= superior mesenteric artery. 


\section{Table II: Laparoscopic findings}

No signs of metastases on laparoscopy

$\mathrm{N}=44(65 \%)$

Metastases:

$\mathrm{N}=24(35 \%)$

liver

$N=5(21 \%)$

peritoneum/ascites

$N=14(58 \%)$

lymph nodes near celiac trunk

$N=3(13 \%)$

liver and peritoneum

$N=2(8 \%)$ 


\section{Legend to the Figures:}

Figure I: Infiltration of the peripancreatic fatplane and $180^{\circ}$ encasement of the superior mesenteric artery (red) by tumour (blue).

Figure II: Thrombus (green) located in the portal vein (red).

Figure III: Overall survival in true locally advanced pancreatic cancer after chemoradiotherapy vs. observation. 


\section{References}

1. Jemal, A., et al., Cancer statistics, 2007. CA Cancer J Clin, 2007. 57(1): p. 43-66.

2. $\quad$ Oettle, H., et al., Adjuvant chemotherapy with gemcitabine vs observation in patients undergoing curative-intent resection of pancreatic cancer: a randomized controlled trial. Jama, 2007. 297(3): p. 267-77.

3. Neoptolemos, J.P., et al., Adjuvant chemoradiotherapy and chemotherapy in resectable pancreatic cancer: a randomised controlled trial. Lancet, 2001. 358(9293): p. 1576-85.

4. Beger, H.G., et al., Intraarterial adjuvant chemotherapy after pancreaticoduodenectomy for pancreatic cancer: significant reduction in occurrence of liver metastasis. World J Surg, 1999. 23(9): p. 946-9.

5. Buchs, N.C., et al., Vascular invasion in pancreatic cancer: evaluation of endoscopic ultrasonography, computed tomography, ultrasonography, and angiography. Swiss Med Wkly, 2007. 137(19-20): p. 28691.

6. Liu, R.C. and L.W. Traverso, Diagnostic laparoscopy improves staging of pancreatic cancer deemed locally unresectable by computed tomography. Surg Endosc, 2005. 19(5): p. 638-42.

7. Traverso, L.W., Pancreatic cancer: surgery alone is not sufficient. Surg Endosc, 2006. 20 Suppl 2: p. S446-9.

8. $\quad$ Butturini, G., et al., The role of laparoscopy in advanced pancreatic cancer diagnosis. Dig Surg, 2007. 24(1): p. 33-7.

9. Doucas, H., et al., Assessment of pancreatic malignancy with laparoscopy and intraoperative ultrasound. Surg Endosc, 2007. 21(7): p. 1147-52.

10. Thomson, B.N., et al., Refining the role of laparoscopy and laparoscopic ultrasound in the staging of presumed pancreatic head and ampullary tumours. Br J Cancer, 2006. 94(2): p. 213-7.

11. Tilleman, E.H., et al., Limitation of diagnostic laparoscopy for patients with a periampullary carcinoma. Eur J Surg Oncol, 2004. 30(6): p. 658-62.

12. Nieveen van Dijkum, E.J., et al., Laparoscopic staging and subsequent palliation in patients with peripancreatic carcinoma. Ann Surg, 2003. 237(1): p. 66-73.

13. Liu, R.C. and L.W. Traverso, Laparoscopic staging should be used routinely for locally extensive cancer of the pancreatic head. J Gastrointest Surg, 2004. 8(8): p. 923-4.

14. Shoup, M., et al., Is there a role for staging laparoscopy in patients with locally advanced, unresectable pancreatic adenocarcinoma? J Gastrointest Surg, 2004. 8(8): p. 1068-71.

15. Stefanidis, D., et al., The current role of staging laparoscopy for adenocarcinoma of the pancreas: a review. Ann Oncol, 2006. 17(2): p. 189-99.

16. Goldstein, D., et al., Gemcitabine with a specific conformal 3D 5FU radiochemotherapy technique is safe and effective in the definitive management of locally advanced pancreatic cancer. Br J Cancer, 2007. 97(4): p. 464-71.

17. Haddock, M.G., et al., Gemcitabine, cisplatin, and radiotherapy for patients with locally advanced pancreatic adenocarcinoma: results of the North Central Cancer Treatment Group Phase II Study N9942. J Clin Oncol, 2007. 25(18): p. 2567-72.

18. Sultana, A., et al., Systematic review, including meta-analyses, on the management of locally advanced pancreatic cancer using radiation/combined modality therapy. Br J Cancer, 2007. 96(8): p. 1183-90.

19. Jeekel, J. and A.D. Treurniet-Donker, Treatment perspectives in locally advanced unresectable pancreatic cancer. Br J Surg, 1991. 78(11): p. 1332-4.

20. Loyer, E.M., et al., Vascular involvement in pancreatic adenocarcinoma: reassessment by thin-section CT. Abdom Imaging, 1996. 21(3): p. 202-6.

21. Phoa, S.S., et al., Value of CT criteria in predicting survival in patients with potentially resectable pancreatic head carcinoma. J Surg Oncol, 2005. 91(1): p. 33-40.

22. Boeck, S., et al., Capecitabine plus oxaliplatin (CapOx) versus capecitabine plus gemcitabine (CapGem) versus gemcitabine plus oxaliplatin ( $m G e m O x)$ : final results of a multicenter randomized phase II trial in advanced pancreatic cancer. Ann Oncol, 2007.

23. Moore, M.J., et al., Erlotinib plus gemcitabine compared with gemcitabine alone in patients with advanced pancreatic cancer: a phase III trial of the National Cancer Institute of Canada Clinical Trials Group. J Clin Oncol, 2007. 25(15): p. 1960-6.

24. Sultana, A., et al., Meta-analyses of chemotherapy for locally advanced and metastatic pancreatic cancer. J Clin Oncol, 2007. 25(18): p. 2607-15.

25. Isacoff, W.H., et al., Phase II trial of infusional fluorouracil, leucovorin, mitomycin, and dipyridamole in locally advanced unresectable pancreatic adenocarcinoma: SWOG S9700. J Clin Oncol, 2007. 25(13): p. 1665-9. 
26. Park, J.K., et al., Gemcitabine chemotherapy versus 5-fluorouracil-based concurrent chemoradiotherapy in locally advanced unresectable pancreatic cancer. Pancreas, 2006. 33(4): p. 397402.

27. Cohen, S.J., et al., A randomized phase III study of radiotherapy alone or with 5-fluorouracil and mitomycin-C in patients with locally advanced adenocarcinoma of the pancreas: Eastern Cooperative Oncology Group study E8282. Int J Radiat Oncol Biol Phys, 2005. 62(5): p. 1345-50.

28. Mishra, G., et al., Phase II trial of induction gemcitabine/CPT-11 followed by a twice-weekly infusion of gemcitabine and concurrent external beam radiation for the treatment of locally advanced pancreatic cancer. Am J Clin Oncol, 2005. 28(4): p. 345-50.

29. Bouvy, N.D., et al., Laparoscopic surgery is associated with less tumour growth stimulation than conventional surgery: an experimental study. Br J Surg, 1997. 84(3): p. 358-61.

30. Shiromizu, A., et al., Effect of laparotomy and laparoscopy on the establishment of lung metastasis in a murine model. Surgery, 2000. 128(5): p. 799-805. 


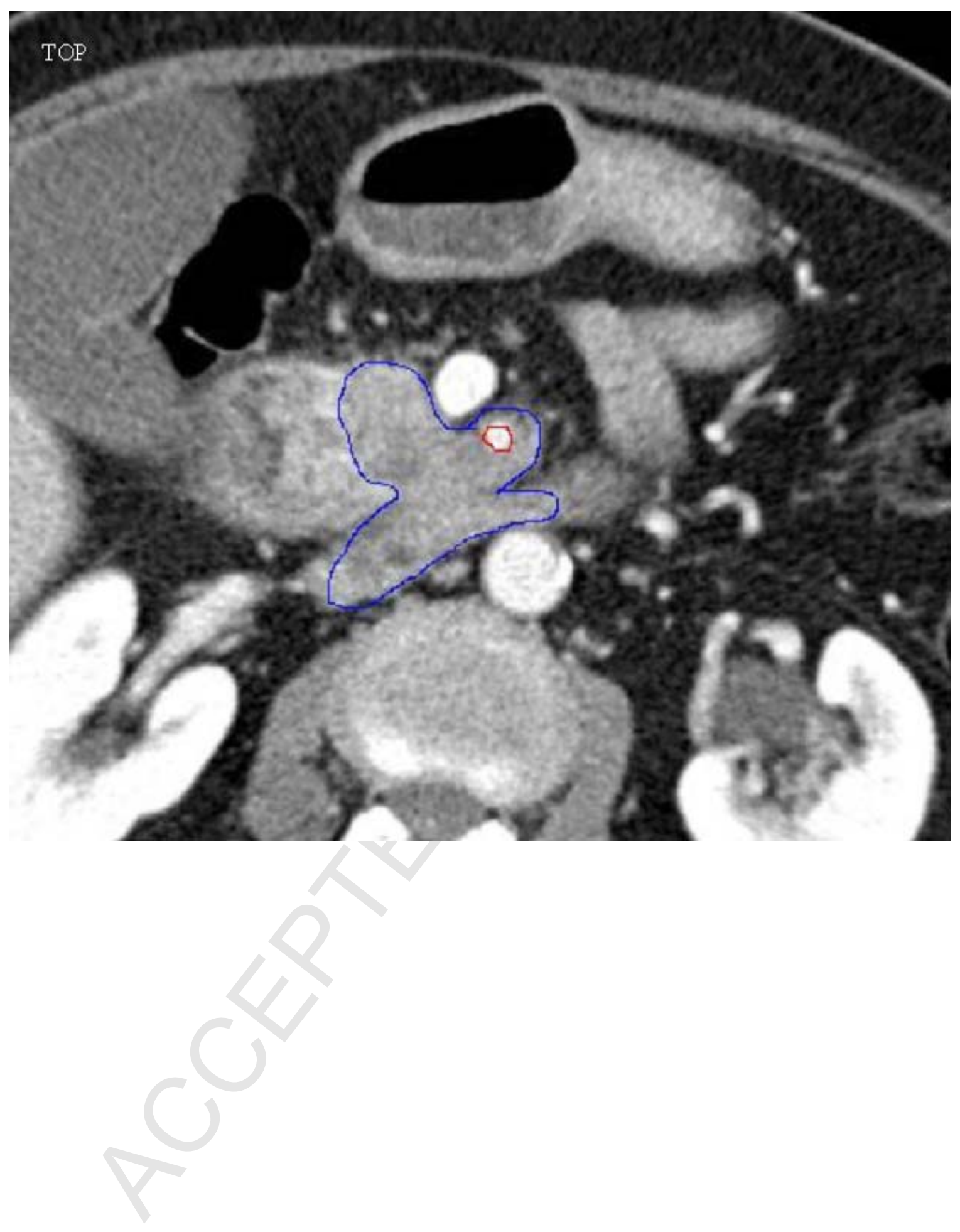




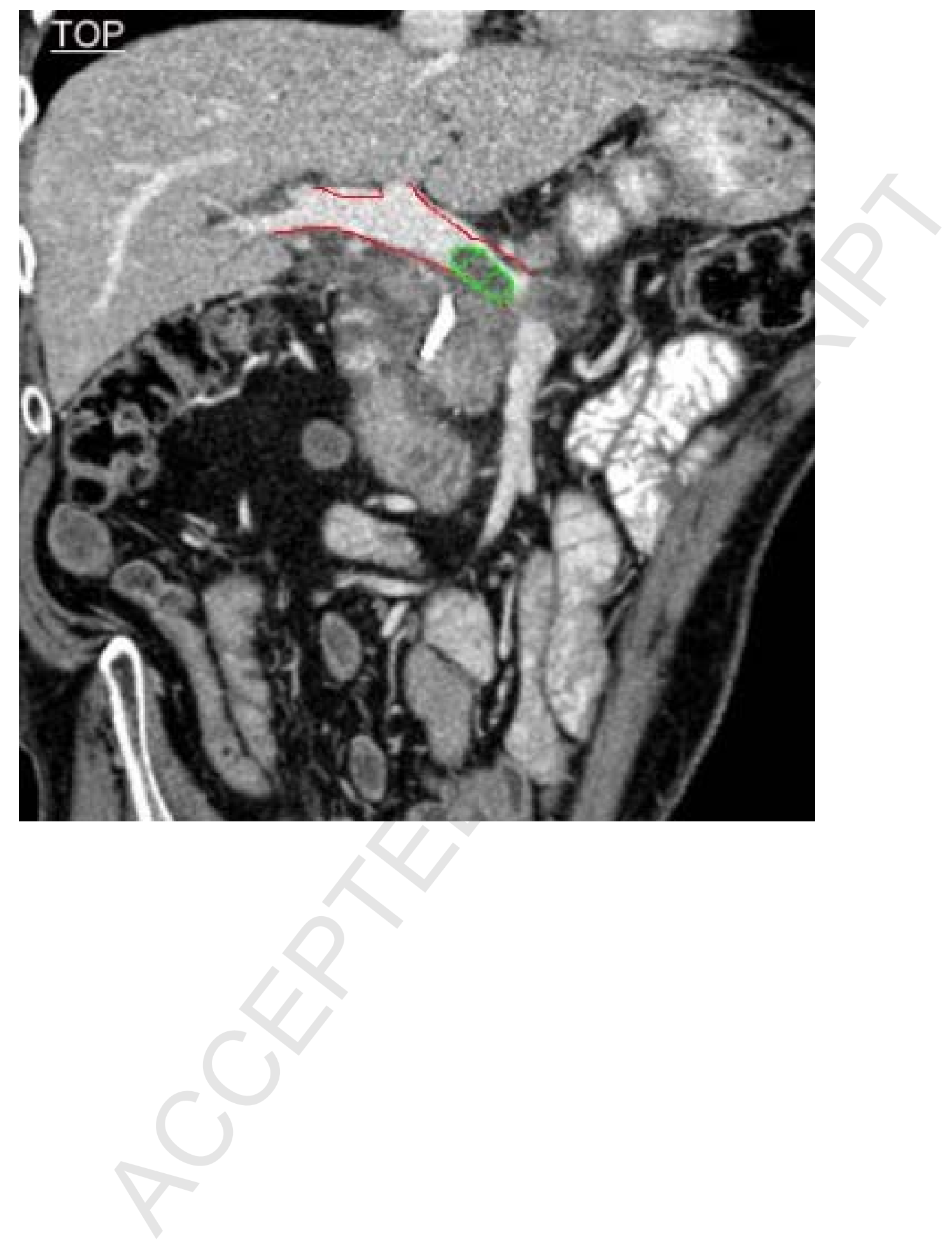




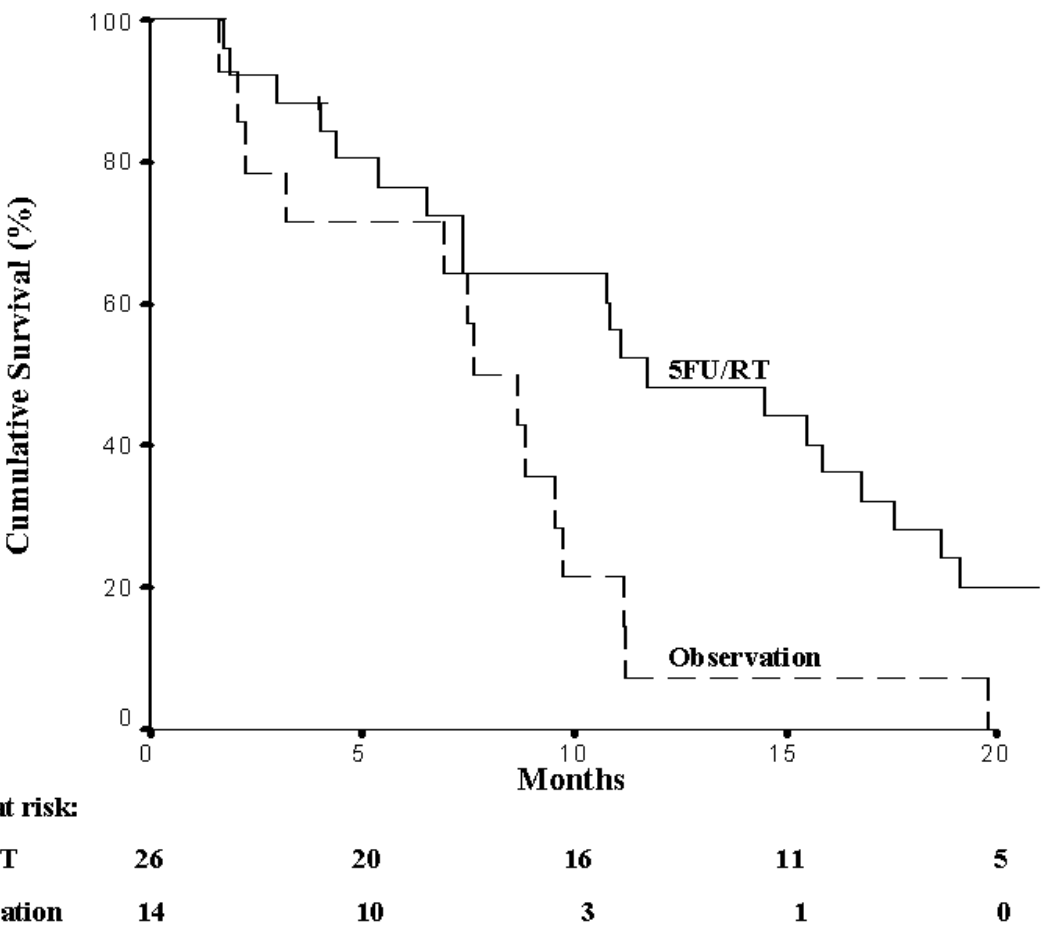

